\title{
Multinozzle Emitter Arrays for Nanoelectrospray Mass Spectrometry
}

\author{
Pan Mao ${ }^{1,4}$, Hung-Ta Wang ${ }^{3,4}$, Peidong Yang ${ }^{2,3}$, \& Daojing Wang ${ }^{1, *}$ \\ ${ }^{1}$ Life Sciences Division, ${ }^{2}$ Materials Sciences Division, Lawrence Berkeley National Laboratory, \\ Berkeley, California \\ ${ }^{3}$ Department of Chemistry, University of California, Berkeley, California
}

${ }^{4}$ These authors contributed equally to this work.

* Correspondence should be addressed to:

Daojing Wang, Ph.D.

Life Sciences Division

Lawrence Berkeley National Laboratory

1 Cyclotron Road, MS 977

Berkeley, CA 94720

Email: djwang@lbl.gov

Fax: 510-495-2535 


\section{DISCLAIMER}

This document was prepared as an account of work sponsored by the United States Government. While this document is believed to contain correct information, neither the United States Government nor any agency thereof, nor the Regents of the University of California, nor any of their employees, makes any warranty, express or implied, or assumes any legal responsibility for the accuracy, completeness, or usefulness of any information, apparatus, product, or process disclosed, or represents that its use would not infringe privately owned rights. Reference herein to any specific commercial product, process, or service by its trade name, trademark, manufacturer, or otherwise, does not necessarily constitute or imply its endorsement, recommendation, or favoring by the United States Government or any agency thereof, or the Regents of the University of California. The views and opinions of authors expressed herein do not necessarily state or reflect those of the United States Government or any agency thereof or the Regents of the University of California. 
Mass spectrometry (MS) is the enabling technology for proteomics and metabolomics. However, dramatic improvements in both sensitivity and throughput are still required to achieve routine MS-based single cell proteomics and metabolomics. Here, we report the silicon-based monolithic multinozzle emitter array (MEA), and demonstrate its proof-of-principle applications in high-sensitivity and high-throughput nanoelectrospray mass spectrometry. Our MEA consists of 96 identical 10-nozzle emitters in a circular array on a 3-inch silicon chip. The geometry and configuration of the emitters, the dimension and number of the nozzles, and the micropillar arrays embedded in the main channel, can be systematically and precisely controlled during the microfabrication process. Combining electrostatic simulation and experimental testing, we demonstrated that sharpened-end geometry at the stem of the individual multinozzle emitter significantly enhanced the electric fields at its protruding nozzle tips, enabling sequential nanoelectrospray for the high-density emitter array. We showed that electrospray current of the multinozzle emitter at a given total flow rate was approximately proportional to the square root of the number of its spraying-nozzles, suggesting the capability of high MS sensitivity for multinozzle emitters. Using a conventional Z-spray mass spectrometer, we demonstrated reproducible MS detection of peptides and proteins for serial MEA emitters, achieving sensitivity and stability comparable to the commercial capillary emitters. Our robust silicon-based MEA chip opens up the possibility of a fully-integrated microfluidic system for ultrahigh-sensitivity and ultrahighthroughput proteomics and metabolomics.

Single Cell Omics unifies biology and technology and has become a new frontier. ${ }^{1}$ For mass spectrometry (MS)-based single cell proteomics and metabolomics, proof-of-principle experiments have been performed to characterize peptides and metabolites using matrix-assisted laser desorption ionization (MALDI)-MS ${ }^{2-4}$ and electrospray ionization (ESI)-MS. ${ }^{5,6}$ However, samples were processed 
individually offline and coverage of proteome and metabolome was limited in these studies. Key challenges still remain. First, further improvement in detection sensitivity; Second, extremely-efficient processing of minute amount of samples, down to a single cell; Third, high-throughput analysis in a cost-effective manner so that a large number of individual cells can be analyzed to achieve statistical significance. Since ESI-MS, ${ }^{7}$ particularly nano-ESI-MS, ${ }^{8}$ is the dominant soft ionization method for analyzing peptides and proteins, a fully-integrated microfluidic front-end system interfaced with nanoESI-MS may serve as a unified platform to address the above-mentioned challenges. Microfluidics enables efficient sample manipulation and processing down to the picoliter even femtoliter range. ${ }^{9}$ Furthermore, the robustness and adaptability of microfabrication processes enables production of massively-parallel functional modules on a single chip for high-throughput analysis.

In fact, one of the actively-pursued areas in MS has been to implement the high-quality interface between microchips and mass spectrometers. ${ }^{10}$ Emitters based on polymeric materials, ${ }^{11-15}$ glass, ${ }^{16,17}$ and silicon using out-of-plane processes, ${ }^{18}$ had been fabricated. However, hydrophobic polymers have inherently undesirable properties for electrospray, such as a strong affinity to proteins and peptides and incompatibility with certain organic solvents; glass substrates are difficult to fabricate for complex structures; and out-of-plane strategy is critically limited in producing monolithically-integrated devices. Efforts in the field have led to two commercial MS-chips: Agilent's HPLC-chip made of polyimide and Waters' "nanoTile" chip made of ceramic. However, these devices have been developed for routine liquid chromatography (LC)-MS/MS applications and lack high-throughput capabilities. Their wide adoption by the research community remains to be seen because of their high costs and requirements for vendor-designated mass spectrometers.

Performing high-throughput ESI-MS remains a challenge because MS itself has a high capital and operational cost, limiting its scalability. Furthermore, MS is a serial detection system typically capable 
of analyzing one sample at a time. Hence, there is a tremendous demand in developing high-throughput MS front-end systems. One approach is to implement multiple LC systems in parallel that are coupled to a single MS detector. This reduces MS down time during sample injection and loading, and hence improves MS usage efficiency. Although in its infancy, the multiple-sprayer platform has been recognized as a potential high-quality interface for high-sensitivity and high-throughput ESI-MS. ${ }^{19}$ "Simultaneous multiple electrosprays" had been achieved with a bundle of fused silica capillaries ${ }^{20}$ and photonic fibers ${ }^{21}$ to improve MS sensitivity. However, the former has a size in the range of millimeters to centimeters and is not suitable for conventional mass spectrometers. Furthermore, neither of them is amenable for monolithic integration on a microchip. "Sequential multiple electrosprays" using multichannel, ${ }^{22,}{ }^{23}$ multitrack, ${ }^{24}$ out-of-plane multiple nozzles, ${ }^{18}$ and gated multi-inlets, ${ }^{25}$ had been implemented for high-throughput MS. In this approach, each sample is processed by a different frontend system (e.g., LC or CE) connected to an individual sprayer. This eliminates sample crosscontamination and allows efficient coupling between various components to reduce the dead volume/time. However, these devices also have intrinsic limitations in monolithic integration.

We had previously developed microfabricated monolithic multinozzle emitters ( $\mathrm{M}^{3}$ emitters) for nanoelectrospray mass spectrometry. ${ }^{26}$ Our in-plane strategy allows ease and flexibility in design, integration, and interfacing to MS. However, the high operating voltage $(\geq 4.5 \mathrm{kV})$ required even for the low-nozzle-number $\mathrm{M}^{3}$ emitters (up to 5 nozzles) to achieve stable electrospray remained problematic. This prevented us from implementing high-nozzle-number $\mathrm{M}^{3}$ emitters (>10 nozzles). Herein, we report a novel approach to create monolithic multinozzle emitter arrays (MEAs) for nanoelectrospray mass spectrometry. We demonstrate two key technical breakthroughs in these devices. First, high-density (up to 96) emitters were constructed in a circular array format on a 3-inch silicon chip (i.e., MEA chip), utilizing the concept of "sequential multiple electrosprays" and hence enabling high-throughput 
applications. Second, sharpened-end emitters with a large number of nozzles (up to 40) per emitter were engineered on the MEA chip, utilizing the concept of "simultaneous multiple electrosprays" and hence enabling high-sensitivity MS detections. We further demonstrate the applicability of our MEA chips for metabolomics and proteomics applications via MS analyses of peptides and tryptic digests.

\section{EXPERIMENTAL SECTION}

\section{Design and fabrication of MEA chips}

MEA chips were designed using the L-Edit software (v15, Tanner Research Inc.). The procedures to fabricate the MEA chips were improved from those for $\mathrm{M}^{3}$ emitters ${ }^{26}$ and involved 9 major steps (Supplement Fig. 1a-i). First, we performed standard photolithography and deep reactive ion etching (DRIE) to pattern and produce channels (with micropillar arrays if needed) and emitters on a 4-inch silicon wafer (a-c). Then, we performed second-layer photolithography and DRIE to define and create access holes with a second film mask (d). Next, we performed thermal fusion bonding between the patterned wafer and another clean wafer (e), followed by wet oxidation to grow a thick oxide of $\sim 1 \mu \mathrm{m}$ on all silicon surfaces including the sealed channels/emitters (f). Afterwards, we performed another photolithography and through-wafer etching steps to sharpen the emitters (left- and right-side, Fig. 1 and Fig. 2) and release the chip from the wafer (g). Subsequently, we sharpened the other two sides (top and bottom, Fig. 1 and Fig. 2) of the emitters by mechanically polishing the emitter stem with the sand paper (h). Finally, we etched away silicon at the sharpened end of the emitters by selective $\mathrm{XeF}_{2}$ etching (i). This final step ended up with protruding nozzles made of $\mathrm{SiO}_{2}$. The fabricated devices were examined by optical microscopy using a Reichert-Jung Polylite 88 microscope (Reichert Microscope Services), and by scanning electron microscopy using a JEOL 6340F FEG-SEM (JEOL Ltd.). 


\section{Electrostatic simulations of MEA emitters}

The multiphysics modeling and simulation software COMSOL (v4.1, COMSOL Inc.) was used to simulate electric fields of 10-nozzle MEA emitters with flat-end, two-side sharpened-end, and four-side sharpened-end features. For simplicity, we did not take into account the presence of complex dynamic gas/fluid behaviors during the actual electrospray process, and only considered static electric fields on MEA emitters relative to the Z-spray sample cone of the Q-TOF API US mass spectrometer (Waters Corp.). Furthermore, we simulated a quadrant instead of the whole device to reduce the dimensions of modeling. The 3D geometry was constructed with the parameters similar to the actual experimental setup. Calculated electric fields were analyzed by 3D slice plots on the central plane $(\mathrm{z}=0)$. The detailed simulation parameters are provided in the Supporting Information.

\section{Electrospray current measurement of MEA emitters}

Total electrospray currents were measured using the Keithley 6487 Picoammeter with built-in data acquisition capabilities (Keithley Instruments). The experimental setup is shown in Supplement Fig. 3

and similar to what had been described. ${ }^{27}$ Electrospray images were taken using a Waters nanoflow camera kit equipped with a MLH-10× microscope (Computar), and using a digital camera Nikon 3700 (Nikon Inc.) mounted on a 6×16 monocular (Specwell Corp.).

\section{Nanoelectrospray mass spectrometry}

All electrospray MS experiments were performed on a hybrid quadrupole/orthogonal Q-TOF API US mass spectrometer (Waters Corp.) as described. ${ }^{28}$ The MEA chip was mounted on the voltage stand and manually rotated every 3-4 degrees for each adjacent emitter. MEA chips were connected with fused silica capillaries (o.d. $\sim 360 \mu \mathrm{m}$, i.d. $\sim 100 \mu \mathrm{m}$ ) by polytetrafluoroethylene (PTFE) tubing whose outer 
diameter (o.d.) matches the i.d. of access holes. Torr Seal epoxy (Agilent Technologies) was applied to permanently seal the connection which could withstand a pressure of more than $100 \mathrm{psi}$. An aluminum conductive tape $(3 \mathrm{M})$ provided the direct electric contact between the voltage stand and the conductive Si-based MEA chip.

\section{Liquid chromatography-MS/MS}

LC-MS/MS analysis was performed using a capillary liquid chromatography system (CapLC) (Waters Corp.) interfaced with a Q-TOF API US mass spectrometer as described. ${ }^{28}$ LC runs using the same C18 column were performed sequentially and independently for individual multinozzle emitters. Peptides eluted from the column were directed through a connecting PTFE tubing (i.d. $\sim 75 \mu \mathrm{m}$, o.d. $\sim$ $1.6 \mathrm{~mm}$ ) to the Picotips or MEA emitters for nanoelectrospray mass spectrometry. Mass spectra were processed using the MassLynx 4.0 SP4 software. Proteins were identified by Mascot (http://www.matrixscience.com) using the MS/MS peak lists exported from the MassLynx.

More experimental details including safety considerations are described in Supporting Information. 


\section{RESULTS AND DISCUSSIONS}

\section{Multinozzle emitter arrays}

We developed MEAs consisting of 96 identical multinozzle emitters in a circular array, uniformly distributed on the periphery of a 3-inch silicon chip (Fig. 1a and 1b). We designed the array layout and inter-emitter spacing in such a way to achieve the best electrospray performance possible while maximizing the number of emitters on the device. Notably, our fabrication processes are amendable for higher-number emitter arrays, e.g., 384 emitters on 6-inch Si wafers.

We utilized sharpened-end features at the four edges (left, right, top, and bottom) of each emitter in order to obtain enhanced electric fields (discussed in the next section). Each emitter was connected to off-chip components via capillaries through its access hole. This is a straightforward design compatible with majority of the microfluidic systems. Fig. 1b shows a representative optical image of a MEA chip with 96 emitters individually connected to capillaries for sample injection. Fig. 1c, 1d, and 1e show SEM images for three adjacent emitters, a single 10-nozzle emitter, and the micropillar arrays monolithically imbedded in the main channel of each emitter, respectively. The micropillar arrays will be utilized in the future for online digestion (e.g., with trypsin coating) or separation (e.g., with C18 or C4 coating) after surface derivatization using silylation chemistry.

We fabricated emitters with varied nozzle numbers, cross-sections, and wall-thickness. Supplement Figure 2 shows three representative sharpened-end emitters with a nozzle cross-section ranging from 10 $\mu \mathrm{m} \times 10 \mu \mathrm{m}$ to $2 \mu \mathrm{m} \times 2.5 \mu \mathrm{m}$, and the corresponding nozzle number per emitter from 1 to 40 . We were able to fabricate nozzles with a cross-section down to $\sim 800 \mathrm{~nm} \times 800 \mathrm{~nm}$ (data not shown), but a significant increase of back pressure ${ }^{29}$ prevented us from utilizing these small nozzles for ESI-MS (see

Supporting Information for more discussion). We have mainly examined the performance of 
multinozzle emitters with a nozzle cross-section of $\sim 10 \mu \mathrm{m} \times 10 \mu \mathrm{m}$ in this work. The potential of using smaller nozzles will be explored in the future.

We achieved a fabrication yield close to $100 \%$ for MEA emitters due to the significantly-improved microfabrication processes. For our $\mathrm{M}^{3}$ emitters, sealed main channels were opened up by mechanical sawing, which resulted in serious clogging. ${ }^{26}$ The fabrication of MEA emitters did not involve the mechanical dicing step. Instead, main channels were opened up by deep reactive ion etching, which is a dry etching process that does not introduce any particles into the main channels. The mechanical polishing step with the sand paper (Step h) typically generates particles larger than the nozzle sizes (e.g., $10 \mu \mathrm{m})$, which are removed by piranha cleaning. Therefore, our new procedures dramatically reduced channel clogging and improved device yields. Additionally, the connection between the MEA chip and outside liquid sources can be improved, by building a custom-made manifold which mechanically assembles the chip with tubings, O-rings, and/or gaskets. This manifold can withstand high pressures and be reused, and also minimizes dead volumes.

\section{Electric fields on the multinozzle emitter arrays}

We utilized a 3D electrostatic simulator to examine the effects of sharpened-end features on the electric fields of emitters. Three types of emitters with the same number of nozzles (i.e., 10) including those for flat-end (Fig. 2a), two-side sharpened-end (Fig. 2b), and four-side sharpened-end (Fig. 2c), were compared. The electric fields were much stronger at the nozzle tips than in the other regions of the emitters for all three cases. But the maximum of the electric fields at the nozzle tips significantly increased from $3.7 \times 10^{6} \mathrm{~V} / \mathrm{m}$ for the flat-end emitters to $6.6 \times 10^{6} \mathrm{~V} / \mathrm{m}$ for the four-side sharpened-end emitters. For each emitter, we observed higher electric fields at the tips of the periphery nozzles than those of the interior ones. In particular, there was a gradual increase from the center to the edge of the 
linear nozzle array, with a maximum increase of $48.7 \%$ from the center nozzle $\left(3.9 \times 10^{6}\right)$ to the edge nozzle $\left(5.8 \times 10^{6}\right)$ for the four-side sharpened-end emitters (Fig. 2c(iii) and Fig. 3a). This was due to the linear format of the nozzle array, the position of the nozzle array relative to the ion cone (Z-spray), the nozzle-nozzle interactions (shielding effects), ${ }^{30}$ and the interactions between the emitter stem and the nozzles on the two edges (i.e., the edge effects). Consistently, we observed even higher electric fields at the corner of the nozzles on two edges, i.e., $6.6 \times 10^{6}$ (left corner, not labeled) vs. $5.8 \times 10^{6}$ (center, labeled) for the leftmost nozzle; and $6.1 \times 10^{6}$ (right corner, not labeled) vs. $5.7 \times 10^{6}$ (center, labeled) for the rightmost nozzle, respectively (Fig. 3a). We further confirmed the simulated pattern of the electric fields experimentally. As demonstrated by the electrospray images of a corresponding 10-nozzle MEA emitter, the spray plumes showed a clear edge effect for the outmost nozzles while relative homogeneity among the inner nozzles (Fig. 3b).

Although enhancement of electric fields at sharp tips is a known phenomenon, ours is one of the first examples showing increased electric fields at the nozzles through sharpening the emitter stems instead of the nozzles themselves for Si-based devices. The protruding feature of the nozzles prevented sample wetting on the emitter surface, while the sharpening of the emitter stems ensured sufficient electric fields for Taylor cone formation. It is expected that additional improvement could be achieved by optimizing the shape of the nozzle support (e.g., sharpening angles for four sides), and the 3D layout of the nozzles (e.g., a circular array). The same modeling strategy is applicable for rational design of microfluidic modules.

\section{Electrospray currents of multinozzle emitter arrays}

We compared the total electrospray currents over a wide range of applied voltages and flow rates for 1- and 10-nozzle MEA emitters and Picotips. As shown in Fig. 4a, electrospray current for 1-nozzle 
MEA emitter reached two plateau regions at $\sim 2.3 \mathrm{kV}$ and $\sim 2.7 \mathrm{kV}$, respectively. Concurrently, we observed three electrospray modes including pulsating $(2.3 \mathrm{kV})$, cone-jet $(2.7 \mathrm{kV})$, and multi-jet $(3.3 \mathrm{kV})$ (Fig. 3b), similar to those described for capillary and elastomeric emitters. ${ }^{31,32}$ For 10 -nozzle MEA emitters, the constant-current plateau region (cone-jet mode) was observed at higher voltages of $\sim 3.6 \mathrm{kV}$ (Fig. 4b). However, its "multi-jet" mode was harder to observe due to the multi-spray nature of the multinozzle emitters (Fig. 3c). On the other hand, we observed comparable electrospray currents for 1nozzle MEA emitter and Picotips under same conditions, although there was no clear plateau region for Picotips (data not shown).

We next tested whether our multinozzle emitters followed the square root $\mathrm{n}$ relationship, i.e., the total electrospray current from the multi-electrosprays in the cone-jet mode is proportional to the square root of the number of sprays (nozzles). ${ }^{27,}{ }^{32}$ We measured the dependence between total electrospray current and applied voltages for 1- and 10-nozzle MEA emitters at given total flow rates, shown in Fig. 4a for $0.6 \mu \mathrm{L} / \mathrm{min}$. We then determined the electrospray current for a particular total flow rate at the plateau region corresponding to the cone-jet mode. As shown in Fig. $\mathbf{4 b}$, the electrospray currents from both 1- and 10-nozzle MEA emitters fitted a power of the total flow rate, with the power constant of 0.47 and 0.48 , respectively. This was consistent with the square root relationship between spray currents and total flow rates. Furthermore, at a given total flow rate, the ratio of electrospray currents between 10- and 1-nozzle MEA emitters was calculated to be $2.65 \sim 2.85$ for the total flow rates of $0.2 \sim 0.6$ $\mu \mathrm{L} / \mathrm{min}$ (Fig. 4b insert), which was 10-20\% less than the predicted theoretical ratio of 3.16, i.e., the square root of 10 (nozzles). ${ }^{27,32}$ The discrepancy might be due to the inhomogeneity among the 10 nozzles as exemplified by their electric field distribution (Fig. 3a), as well as the much stronger internozzle interactions for 10-nozzle MEA emitters in comparison to a bundle of multiple capillary emitters ${ }^{32}$. In fact, both the size (a cross-section of $\left.\sim 10 \mu \mathrm{m} \times 10 \mu \mathrm{m}\right)$ and inter-nozzle spacing $(\sim 40 \mu \mathrm{m})$ of MEA 
emitters were significantly smaller than those of the bundle of fused silica capillaries (i.d. $\sim 19 \mu \mathrm{m}$ and the inter-capillary spacing of $\sim 500 \mu \mathrm{m}$, respectively). In addition, there were inter-emitter interactions on MEA chips. Nevertheless, the significant increase of electrospray currents in 10-nozzle MEA emitters suggested the feasibility of achieving even higher MS sensitivity for multinozzle emitters with larger nozzle numbers.

\section{High-throughput mass spectrometry using multinozzle emitter arrays}

We first confirmed that sharpening dramatically reduced operating voltages for ESI-MS using sharpened-end $\mathrm{M}^{3}$ emitters (Supplement Figure 4). Strikingly, optimal operating voltage was observed at $\sim 1.8 \mathrm{kV}$ for single-nozzle emitters, which was similar to those for Picotips $(1.5 \mathrm{kV} \sim 2.3 \mathrm{kV})$, and a

dramatic improvement from that for flat-end single-nozzle emitters $(4.5 \mathrm{kV} \sim 4.8 \mathrm{kV}){ }^{26}$ For a sharpened-end 20-nozzle emitter, the optimal voltage was $3.5 \mathrm{kV}$, confirming the aforementioned internozzle interactions. ${ }^{30}$ We observed that both the optimal voltage and MS sensitivity increased with nozzle numbers. For example, there was an on average $\sim 2$-fold increase in sensitivity for the 20 -nozzle relative to the 1-nozzle emitters. As mentioned above, electrospray current and therefore MS sensitivity was predicted to be proportional to the square root of the number of nozzles. ${ }^{27}$ If this holds for our multinozzle emitters, one would expect about 4.5 -fold increase. The difference was probably due to the suboptimal efficiency of ion collection and transmission by the Z-spray sample cone of our mass spectrometer, because electrosprays from multinozzle emitters were spread out significantly. Future implementation of a funnel-shaped sample cone ${ }^{20}$ may increase MS sensitivity for multinozzle emitters.

We next compared the performance of MEA emitters, free-standing sharpened-end $\mathrm{M}^{3}$ emitters, and Picotips. All three types of emitters achieved comparably high MS sensitivity for $0.1 \mu \mathrm{M}$ GFP (Fig. 5a,). We observed a slightly $(\sim 10 \%)$ higher MS sensitivity for both 10-nozzle MEA and $\mathrm{M}^{3}$ emitters than 
Picotips. Importantly, both 10-nozzle MEA and $\mathrm{M}^{3}$ emitters achieved higher MS sensitivity than their 1nozzle counterparts, validating the value of the multinozzle design. However, the fold change was around 1.5-2.0 and less than what would be expected from the square root $\mathrm{n}$ relationship, i.e., $\sim 3$ fold, as shown by the electrospray current (Fig. 4). As discussed above, future optimization of both the MS ion optics and the relative position between MEA emitters and the ion cone (Fig. 5a insert) may mitigate this limitation. We achieved stable electrosprays at 3.5 and $4.5 \mathrm{kV}$ for 1- and 10-nozzle MEA emitters, respectively, while at 1.8 and $3.2 \mathrm{kV}$ for 1- and 10-nozzle sharpened-end $\mathrm{M}^{3}$ emitters, respectively (Fig. 5a). This was presumably due to the emitter-emitter interactions on the MEA chip, in addition to the nozzle-nozzle interactions within an emitter encountered by both types of emitters. Therefore, an operating voltage higher than the maximum of $5.0 \mathrm{kV}$ provided by our current Q-TOF mass spectrometer, is needed to for MEA emitters with even larger nozzle numbers (e.g., 40). We checked the MS stability for MEA emitters using $0.1 \mu \mathrm{M}$ and $1.0 \mu \mathrm{M}$ GFP, respectively. The relative standard deviation (RSD) for either 1- or 10-nozzle MEA emitters was similar to that of Picotips (Fig. 5b).

We further demonstrated the proof-of-principle applications of MEA emitters in high-throughput proteomics. Fig. 5c shows representative LC-MS/MS chromatograms for 100 fmole tryptic digests of bovine serum albumin (BSA, $\sim 67 \mathrm{kDa}$ ), with one TOF MS (BPI, base peak intensity) each for one Picotip and 7 individual MEA emitters chosen randomly out of the 96 on a MEA chip. There was no significant difference among these chromatograms. In addition, BSA was confidently identified in all cases. With MEA emitters, we obtained on average a Mascot score of 1300 and $43 \%$ sequence coverage with 27 peptides sequenced, while for Picotip emitters a Mascot score of 1333 and $46 \%$ sequence coverage. The small discrepancy among MEA emitters presumably resulted from the slight difference in the positioning of each emitter relative to the ion cone of our mass spectrometer. This can be mitigated in the future through a computer-controlled rotary system optimized for the 3-inch MEA chip. 
Our results demonstrated that MEA emitters could be interfaced with LC-MS/MS for sequential and reproducible high-sensitivity proteomic analyses. It is conceivable that multiple (up to 96) LC systems can be interfaced with our MEA chip to achieve, either sequential (if using only one mass spectrometer) or parallel (if using multiple, for example, miniaturized mass spectrometers ${ }^{33,34}$ ), high-throughput MS analyses. More importantly, our MEA platform is ready for the high-level integration with additional functional modules, to achieve "Lab-on-a-chip". For example, the micropillar arrays embedded in the main channel can be utilized for digestion and separation. The fully-integrated system will dramatically increase the sensitivity and throughput for mass spectrometry-based metabolomics and proteomics, through efficient manipulation and processing of an extremely small amount of samples (such as a single cell), and by diminishing the processing time for cell manipulation, and protein digestion and separation, etc. Our platform can be further interfaced with other technologies such as femtoliter and picoliter-sized droplets for manipulating single cells. ${ }^{35}$ Since our MEA chip is made of conductive Si, there is no liquid junction and conductive coating needed to establish the electric contact between voltage supplies and the chip. This added advantage simplifies the fluidic control on integrated MEA chips. Our design can be extended to MEAs with even higher emitter numbers (e.g., 384), thus enabling "ultrahigh-throughput". With further optimization, our MEA emitters will achieve even better performance in MS sensitivity and stability, thus enabling "ultrahigh-sensitivity".

\section{CONCLUSIONS}

By taking advantages of the maturity and flexibility of silicon microfabrication technologies, we demonstrate massively-parallel multinozzle emitters integrated uniformly in a circular array, enabling high-sensitivity and high-throughput nanoelectrospray mass spectrometry. Our MEA chip is the first silicon-based, robust, and microfabricated monolithic multinozzle emitters implemented in a high- 
throughput array format. Our MEA chip has multiple advantages. Firstly, it eliminates potential sample carryover because each sample will be analyzed by a different emitter. Secondly, it improves reproducibility for parallel analysis because emitters are identically microfabricated. Thirdly, due to the robustness and inert nature of silicon material, MEA chip can work under various conditions and be reused. Fourthly, the clogging at the nozzle tips due to salt and debris built-ups, typically encountered by the commercial Picotips, is significant mitigated due to the multinozzle design. In addition, a series of prefilters can be readily fabricated in the main channels to prevent large debris or particles from entering the nozzles and thereby effectively reduce the clogging. Fifthly, the throughput can be further improved by increasing the wafer size (e.g., from 4- to 6-inch) and optimizing the overall layout geometry. Lastly, the MS sensitivity can be further improved if emitters with an even larger number of nozzles are interfaced with optimized ion optics for efficient ion collection and transmission. In summary, we present the first demonstration of silicon-based monolithic multinozzle emitter arrays (MEAs) for nanoelectrospray mass spectrometry. Once integrated with other functional modules, our MEA chips have the potential to serve as a unified platform for future ultrahigh-sensitivity and ultrahigh-throughput proteomics and metabolomics. 


\section{ACKNOWLEDGMENT}

We thank Dr. Woong Kim and Dr. Zhaoyu Zhang for technical assistance, Dr. Jian Jin for discussion, and UC-Berkeley Marvell Nanofabrication Laboratory for facility access. This work was supported by the Director, Office of Science, of the U.S. Department of Energy under Contract No. DE-AC0205CH11231, and in part by the National Institutes of Health Grant R21GM077870 (to D.W.). 


\section{REFERENCE}

(1) Wang, D.; Bodovitz, S. Trends Biotechnol. 2010, 28, 281-290.

(2) Whittal, R. M.; Keller, B. O.; Li, L. Anal. Chem. 1998, 70, 5344-5347.

(3) Rubakhin, S. S.; Sweedler, J. V. Nat. Protoc. 2007, 2, 1987-1997.

(4) Amantonico, A.; Urban, P. L.; Fagerer, S. R.; Balabin, R. M.; Zenobi, R. Anal. Chem. 2010, 82, 7394-7400.

(5) Lapainis, T.; Rubakhin, S. S.; Sweedler, J. V. Anal. Chem. 2009, 81, 5858-5864.

(6) Shrestha, B.; Vertes, A. Anal. Chem. 2009, 81, 8265-8271.

(7) Fenn, J. B.; Mann, M.; Meng, C. K.; Wong, S. F.; Whitehouse, C. M. Science 1989, 246, 64-71.

(8) Wilm, M.; Mann, M. Anal. Chem. 1996, 68, 1-8.

(9) Whitesides, G. M. Nature 2006, 442, 368-373.

(10) Sikanen, T.; Franssila, S.; Kauppila, T. J.; Kostiainen, R.; Kotiaho, T.; Ketola, R. A. Mass Spectrom. Rev. 2010, 29, 351-391.

(11) Licklider, L.; Wang, X. Q.; Desai, A.; Tai, Y. C.; Lee, T. D. Anal. Chem. 2000, 72, 367-375.

(12) Yang, Y.; Kameoka, J.; Wachs, T.; Henion, J. D.; Craighead, H. G. Anal. Chem. 2004, 76, 25682574.

(13) Kim, J. S.; Knapp, D. R. J. Am. Soc. Mass Spectrom. 2001, 12, 463-469.

(14) Schilling, M.; Nigge, W.; Rudzinski, A.; Neyer, A.; Hergenroder, R. Lab Chip 2004, 4, $220-224$.

(15) Le Gac, S.; Cren-Olive, C.; Rolando, C.; Arscott, S. J. Am. Soc. Mass Spectrom. 2004, 15, 409412.

(16) Hoffmann, P.; Eschner, M.; Fritzsche, S.; Belder, D. Anal. Chem. 2009, 81, 7256-7261.

(17) Mellors, J. S.; Jorabchi, K.; Smith, L. M.; Ramsey, J. M. Anal. Chem. 2010, 82, 967-973.

(18) Schultz, G. A.; Corso, T. N.; Prosser, S. J.; Zhang, S. Anal. Chem. 2000, 72, 4058-4063.

(19) Gibson, G. T.; Mugo, S. M.; Oleschuk, R. D. Mass Spectrom. Rev. 2009, 28, 918-936.

(20) Kelly, R. T.; Page, J. S.; Zhao, R.; Qian, W. J.; Mottaz, H. M.; Tang, K.; Smith, R. D. Anal. Chem. 2008, 80, 143-149.

(21) Su, S.; Gibson, G. T.; Mugo, S. M.; Marecak, D. M.; Oleschuk, R. D. Anal. Chem. 2009, 81, 7281-7287.

(22) Xue, Q.; Foret, F.; Dunayevskiy, Y. M.; Zavracky, P. M.; McGruer, N. E.; Karger, B. L. Anal. Chem. 1997, 69, 426-430.

(23) Liu, H.; Felten, C.; Xue, Q.; Zhang, B.; Jedrzejewski, P.; Karger, B. L.; Foret, F. Anal. Chem. 2000, 72, 3303-3310.

(24) Dayon, L.; Abonnenc, M.; Prudent, M.; Lion, N.; Girault, H. H. J. Mass Spectrom. 2006, 41, 1484-1490.

(25) Moini, M.; Jiang, L.; Bootwala, S. Rapid Commun. Mass Spectrom. 2011, 25, 789-794.

(26) Kim, W.; Guo, M.; Yang, P.; Wang, D. Anal. Chem. 2007, 79, 3703-3707.

(27) Tang, K.; Lin, Y.; Matson, D. W.; Kim, T.; Smith, R. D. Anal. Chem. 2001, 73, 1658-1663.

(28) Wang, D.; Park, J. S.; Chu, J. S.; Krakowski, A.; Luo, K.; Chen, D. J.; Li, S. J. Biol. Chem. 2004, 279, 43725-43734.

(29) Hosokawa, K.; Fujii, T.; Endo, I. Anal. Chem. 1999, 71, 4781-4785.

(30) Tatemoto, Y.; Ishikawa, R.; Takeuchi, M.; Takeshita, T.; Noda, K.; Okazaki, T. Chem. Eng. Technol. 2007, 30, 1274-1279.

(31) Marginean, I.; Kelly, R. T.; Page, J. S.; Tang, K.; Smith, R. D. Anal. Chem. 2007, 79, 8030-8036.

(32) Kelly, R. T.; Tang, K.; Irimia, D.; Toner, M.; Smith, R. D. Anal. Chem. 2008, 80, 3824-3831.

(33) Gao, L.; Song, Q.; Patterson, G. E.; Cooks, R. G.; Ouyang, Z. Anal. Chem. 2006, 78, 5994-6002. 
(34) Malcolm, A.; Wright, S.; Syms, R. R.; Dash, N.; Schwab, M. A.; Finlay, A. Anal. Chem. 2010, 82, 1751-1758.

(35) Chiu, D. T.; Lorenz, R. M. Acc. Chem. Res. 2009, 42, 649-658. 


\section{FIGURE CAPTIONS}

Figure 1 | Multinozzle emitter array chip. (a) A plan view of a 96-emitter array chip. The green circles represent through-holes for sample injection. The red lines represent microfluidic channels of $400 \mu \mathrm{m}$ in width and $10 \mu \mathrm{m}$ in depth, which can be embedded with ordered micropillar arrays. The blue curves represent the sharpened features of the multinozzle emitters. (b) High-definition photograph of a 3-inch MEA chip fabricated from 4-inch silicon wafers. The device is connected to 96 silica capillaries via the

PTFE tubing. (c), (d) SEM images of 10-nozzle emitters with different magnifications. Each emitter consists of a linear 10-nozzle array, with a conduit length of around $100 \mu \mathrm{m}$ and a cross-section of $10 \mu \mathrm{m} \times 10 \mu \mathrm{m}$, protruding out from a hollow silicon sliver. The inter-nozzle spacing is $40 \mu \mathrm{m}$. (e) SEM images of micropillar-arrays within a main channel. The pillars are $10 \mu \mathrm{m}$ deep with a diameter of 4.5 $\mu \mathrm{m}$ and spaced by $5.5 \mu \mathrm{m}$. They are arranged according to an equilateral triangular grid. Boxes in (a) indicate the corresponding zoom-in regions for (c) and (e), respectively.

Figure 2 Simulated electric fields on MEA chips. Three-dimensional electrostatic modeling for representative flat-end (a), two-side sharpened-end (b), and four-side sharpened-end (c) emitters, is shown. Every emitter contains 10 nozzles each with a cross-section of $10 \mu \mathrm{m} \times 10 \mu \mathrm{m}$. The $3 \mathrm{D}$ slice plots of electric fields on the three types of emitters (i), their relative position to the ion cone (ii), and the close-up views on the central emitters designated by the white arrows (iii), are shown. The voltage for the mass spectrometer ion cone and MEA chip is set to be $40 \mathrm{~V}$ and $3000 \mathrm{~V}$, respectively. The maximum magnitude of the electric fields $(\mathrm{V} / \mathrm{m})$ at the nozzle tip of each type of emitters is highlighted on the scale bars individually (iii). 
Figure 3 Electrospray modes on MEA chips. (a) Comparison between electrostatic simulation and electrospray on a MEA chip. (a-i) representative values of the simulated electric fields at the center of each nozzle, on a four-side sharpened-end 10-nozzle MEA emitter. The voltages and the relative geometry for the ion cone and the MEA chip are the same as in Figure 2c. (a-ii) corresponding electrospray images for the 10-nozzle MEA emitter. Electrospray was performed using 50\% methanol $/ \mathrm{H}_{2} \mathrm{O}+0.1 \%$ formic acid at a flow rate of $3.0 \mu \mathrm{L} / \mathrm{min}$. (b) Electrospray images of 1-nozzle MEA emitters at the flow rate of $0.6 \mu \mathrm{L} / \mathrm{min}$, showing the (b-i) pulsating $(2.3 \mathrm{kV})$, (b-ii) cone-jet $(2.7 \mathrm{kV})$, and (b-iii) multi-jet $(3.3 \mathrm{kV})$ modes. (c) Electrospray images of 10-nozzle MEA emitters at the flow rate of $1.0 \mu \mathrm{L} / \mathrm{min}$, showing the (c-i) pulsating, (c-ii) cone-jet, and (c-iii) "multi-jet" modes at indicated voltages. The images were taken using the setup for electrospray current measurements. The solvent was $50 \%$ methanol $/ \mathrm{H}_{2} \mathrm{O}+1 \%$ acetic acid. All nozzles have a cross-section of $10 \mu \mathrm{m} \times 10 \mu \mathrm{m}$. Scale bars in a-c are $500 \mu \mathrm{m}$.

Figure 4 Electrospray currents of MEA emitters. (a) Representative curves of total electrospray currents for 1- and 10-nozzle MEA emitters, as a function of applied voltages at the total flow rate of 0.6 $\mu \mathrm{L} / \mathrm{min}$. The plateau regions of both curves designated by the dashed box indicate the cone-jet mode regimes for electrospray at this flow rate. (b) Comparison of total electrospray currents between 1- and 10-nozzle MEA emitters, spraying in the cone-jet mode regimes at different total flow rates. The values were fitted by a power-law function with the constant of 0.4726 and 0.4832 for 1 - and 10-nozzle emitters, respectively. The inset shows the relative ratios between 10- and 1-nozzle MEA emitters as a function of total flow rates. All nozzles have a cross-section of $10 \mu \mathrm{m} \times 10 \mu \mathrm{m}$. The solvent was $50 \%$ methanol $/ \mathrm{H}_{2} \mathrm{O}+1 \%$ acetic acid. Error bars: s.d., calculated for 3-5 individual emitters. The error bars for 1-nozzle MEA emitters were too small to display. 
Figure 5 High-throughput mass spectrometry using MEA emitters. (a) Comparison of MS sensitivity between different types of emitters. All GFP counts were obtained for $0.1 \mathrm{pmole} / \mu \mathrm{L}$ GFP in $50 / 50$ acetonitrile $/ \mathrm{H}_{2} \mathrm{O}+0.1 \%$ formic acid with a flow rate of $0.6 \mu \mathrm{L} / \mathrm{min}$. The optimal voltages to achieve the stable cone-jet mode spray are designated for each emitter. The insert shows a representative electrospray image for a 10-nozzle MEA emitter and its position relative to the ion cone (left). Error bars: s.d. ( $\mathrm{n} \geq 10$ ). (b) Comparison of stability between Picotips and MEA emitters for 0.1 and 1 pmole/ $\mu \mathrm{L}$ GFP, respectively. Error bars: s.d. (n $\geq 10$ ). (c) Reproducibility of MEA emitters. The base peak intensity (BPI) chromatograms show HPLC gradient elution separation of 100 fmole tryptic digests of bovine serum albumin (BSA) with MS detection for 7 individual 10-nozzle MEA emitters and a Picotip emitter. The tested MEA emitters were randomly chosen from the 96 emitters on a MEA chip. 


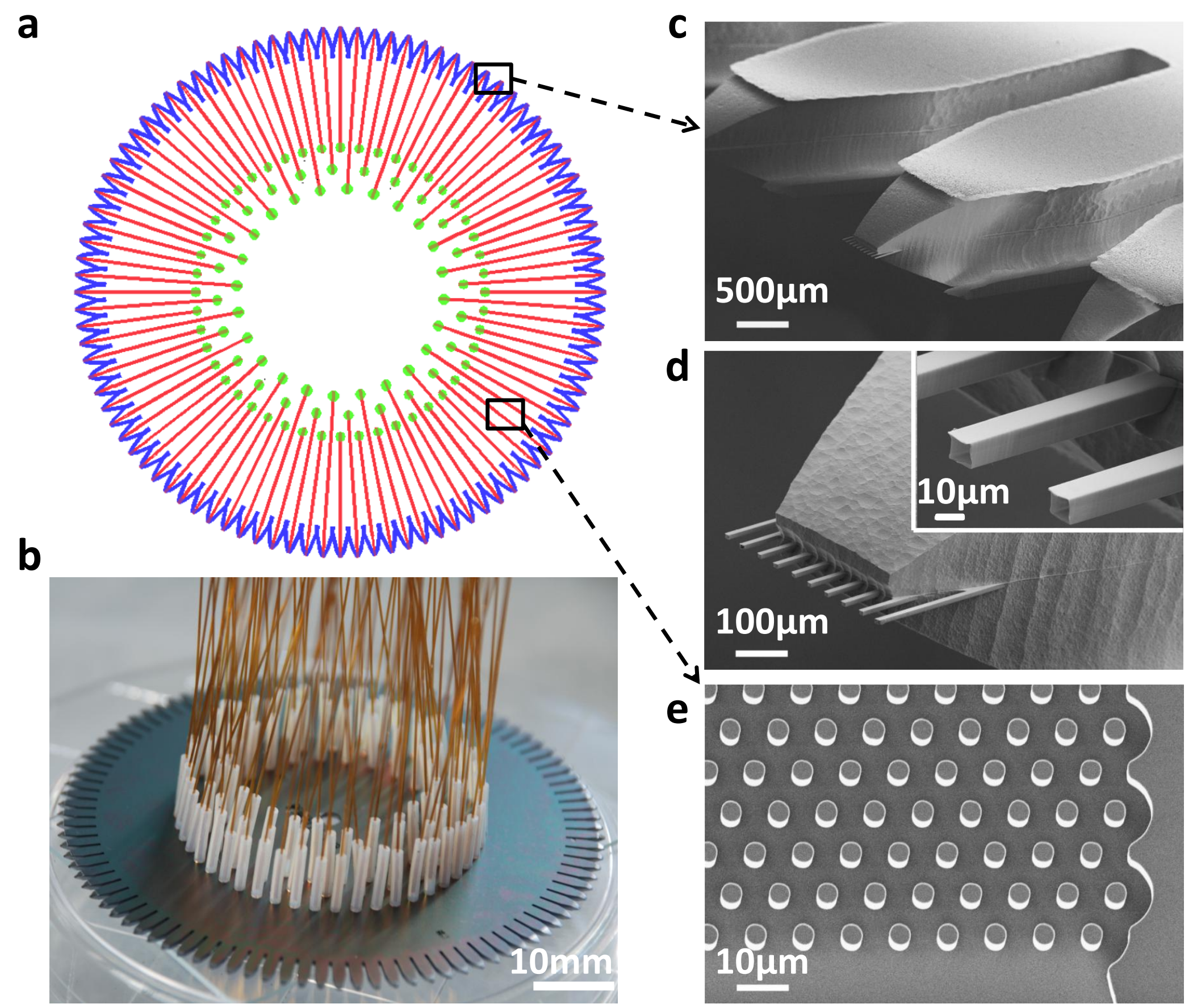




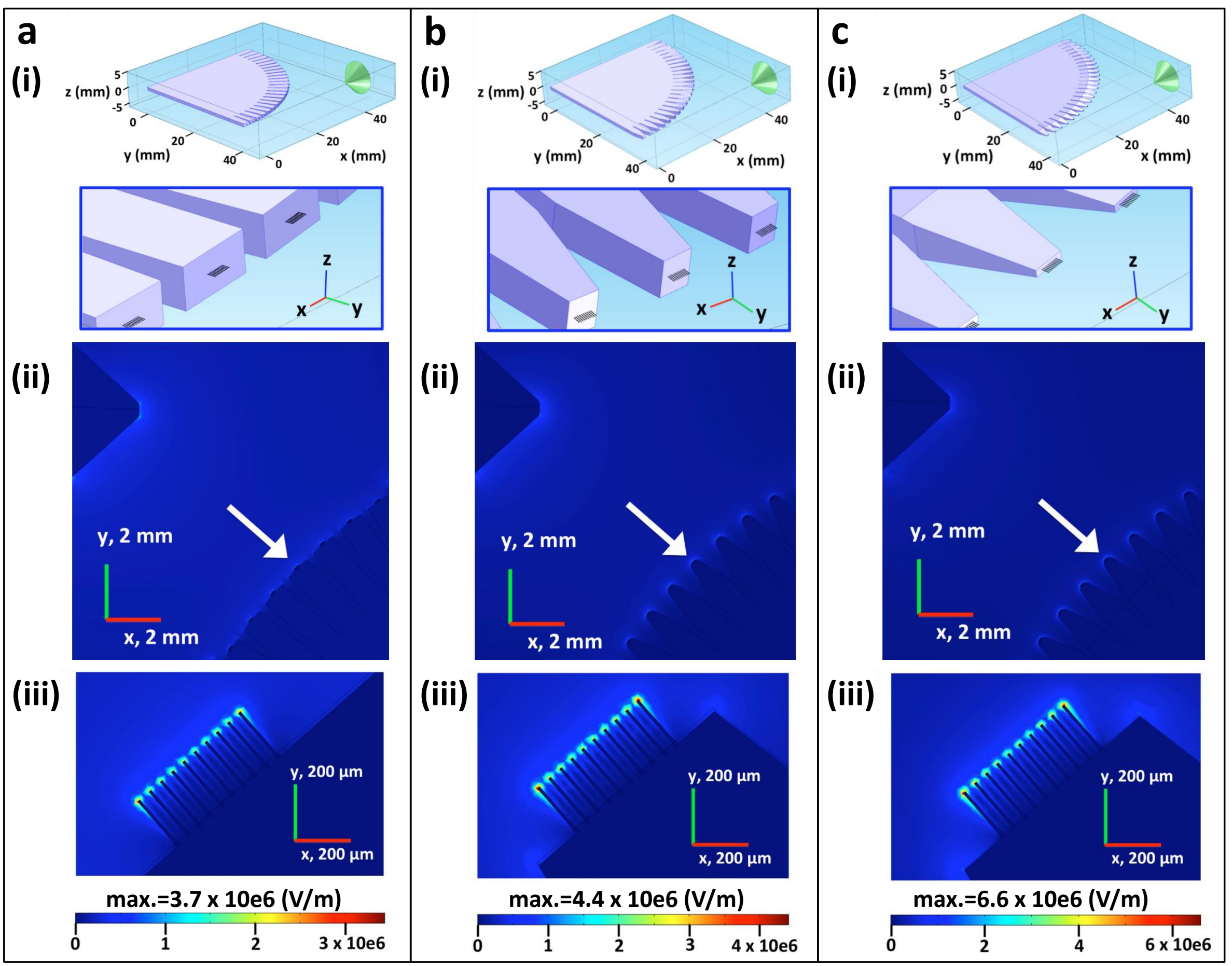


a

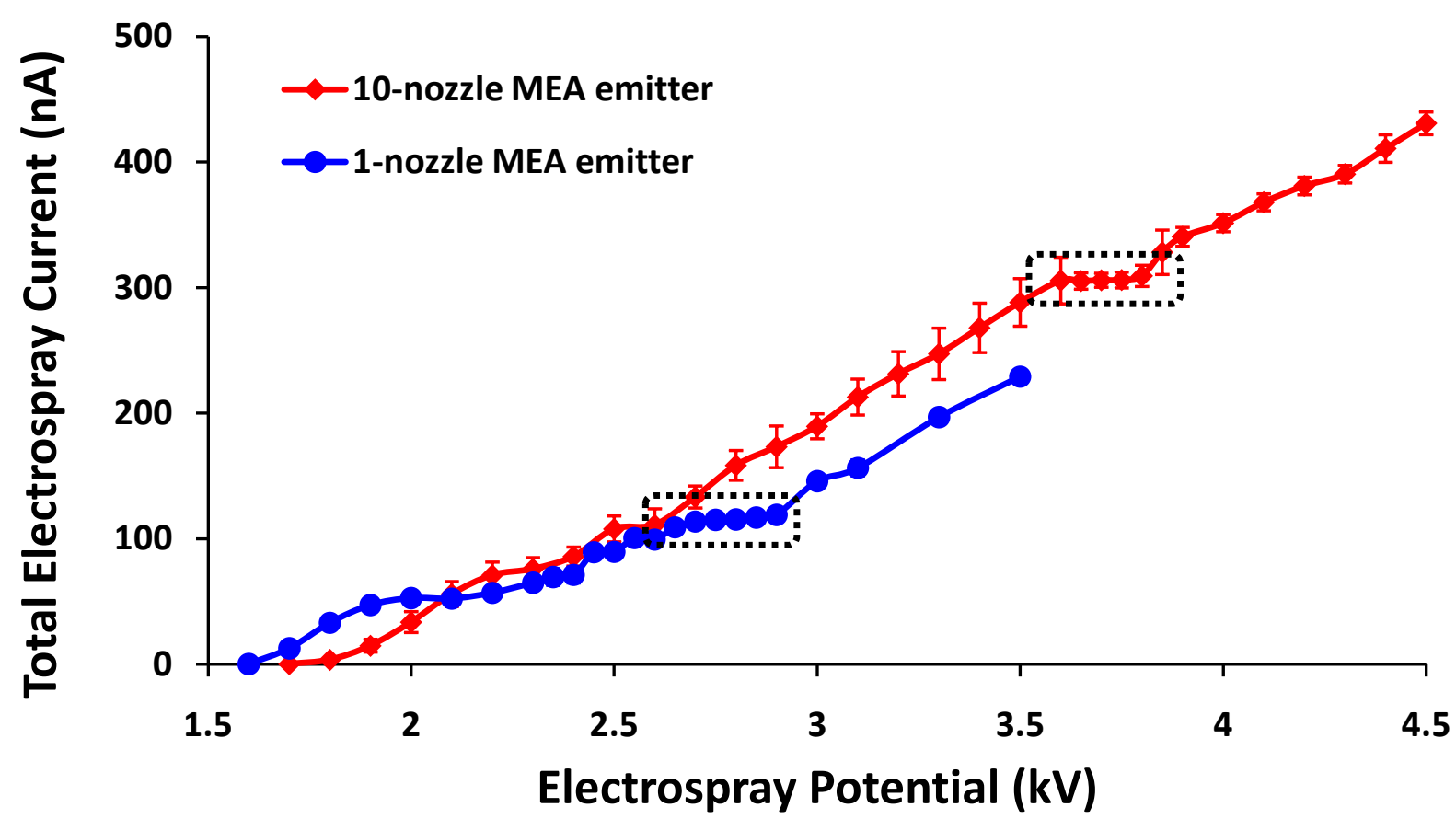

b

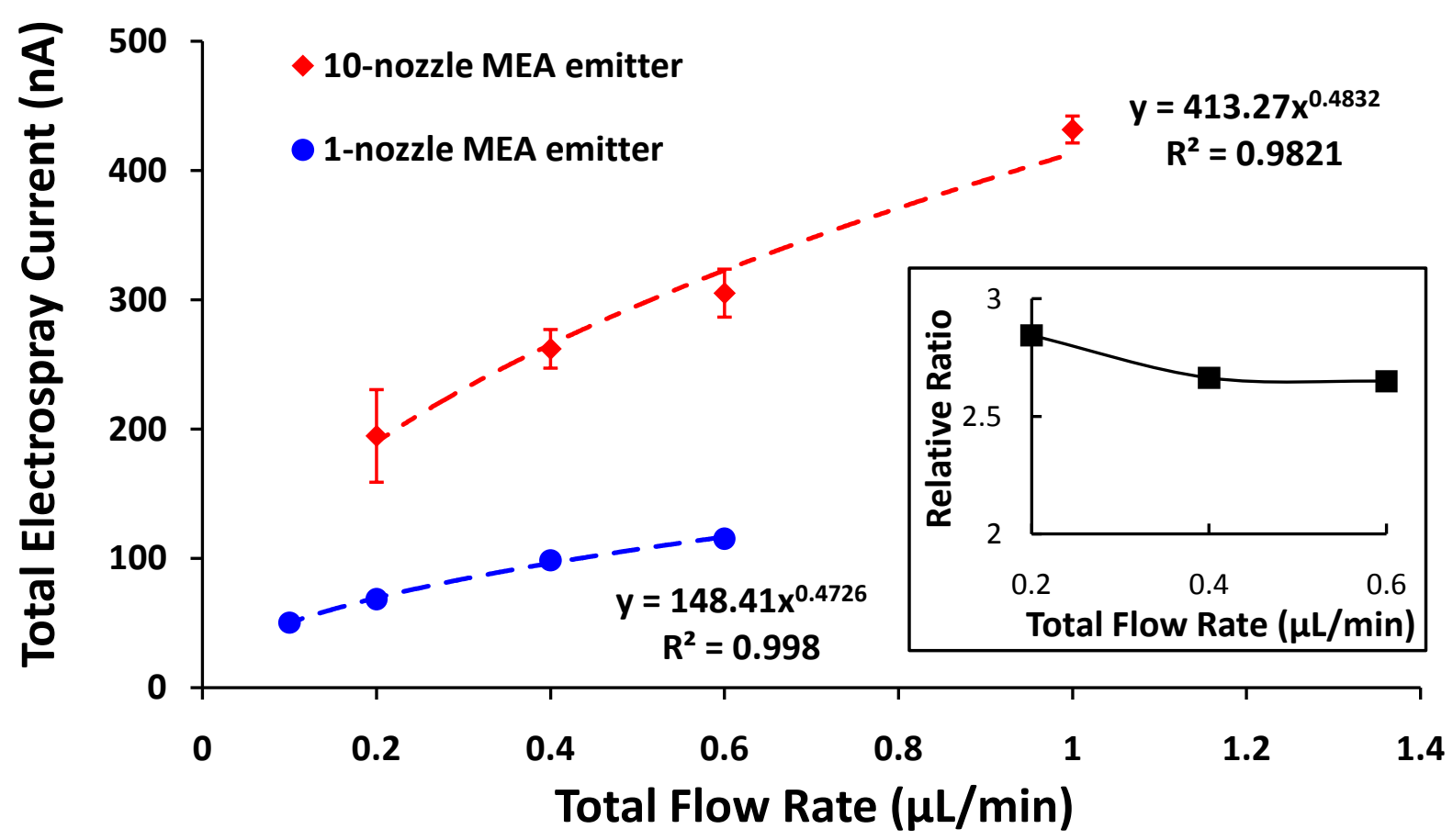


